\title{
Review of: "A Novel Approach Towards Less Invasive Multi “Omics Gut Analyses: A Pilot Study"
}

Subhradip Karmakar

Potential competing interests: The author(s) declared that no potential competing interests exist.

Excellent article, nicely crafted and presented. The information is of prime importance for researcher working in gut microbiome. Does the authors have any suggestion of non-invasive methods for metabolomic studies? Overall a good reference study. 